ESAIM: PROCEEDINGS AND SURVEYS, March 2016, Vol. 53, p. 49-63

M. Campos Pinto and F. Charles, Editors

\title{
TOWARDS AUTOMATED MAGNETIC DIVERTOR DESIGN FOR OPTIMAL HEAT EXHAUST
}

\author{
Maarten Blommaert ${ }^{1}$, Holger Heumann ${ }^{2}$, Martine Baelmans ${ }^{3}$, Nicolas R. \\ GAUGeR $^{4}$ AND Detlev ReIter ${ }^{1}$
}

\begin{abstract}
Avoiding excessive structure heat loads in future fusion tokamaks is regarded as one of the greatest design challenges. In this paper, we aim at developing a tool to study how the severe divertor heat loads can be mitigated by reconfiguring the magnetic confinement. For this purpose, a free boundary equilibrium code is integrated with a plasma edge transport code to work in an automated fashion. Next, a practical and efficient adjoint based sensitivity calculation is proposed to evaluate the sensitivities of the integrated code. The sensitivity calculation is finally applied to a realistic test case and compared with finite difference sensitivity calculations.
\end{abstract}

\section{INTRODUCTION}

In magnetically confined fusion tokamaks, the so-called divertor configuration is currently the most popular confinement concept. Using a specific magnetic divertor configuration with a saddle point in the poloidal magnetic flux function (also known as an X-point), plasma flows are "diverted" to flow to a -for the purpose designed- divertor target structure. In this way, tokamak divertors serve as an exhaust for both particle and heat fluxes in fusion reactors. They are therefore simultaneously responsible for enabling easy helium ash removal and retaining the divertor heat load below material limits. For next step fusion reactors, such as ITER [1] or Demo [2], a well-designed divertor configuration will be crucial to realize a sufficiently long lifetime for plasma-facing components.

In many engineering areas such as aerodynamics or fluid dynamics, automated optimization methods greatly assist in the design of new devices. These automated methods introduce a cost function that penalizes the deviation of the physical system induced by some given design from the design goal and use standard algorithms for constraint optimization problems to find the optimal design.

The basic design variables for divertors are the shape of the divertor structure and its magnetic field configuration that can be altered by the currents in the auxiliary coils. The physical system of interest is the flow in the plasma edge governed by highly complex physical interactions between plasma, neutrals, and solid materials. Numerical simulations of such systems are extremely costly and time consuming (see e.g. the B2-EIRENE plasma edge code system $[1,3])$.

In order to efficiently exploit these simulations for automated divertor design efficient optimal design methods should be applied. Therefore we resort to optimization methods that use adjoints to compute the gradients of the cost function. This approach avoids that the computational cost of the gradient computation scales with the number of design variables. Recently, these adjoint based optimization methods were combined with tokamak

corresponding author e-mail: m.blommaert@fz-juelich.de

1 Institute of Energy and Climate Research (IEK-4), FZ Jülich GmbH, D-52425 Jülich, Germany

2 TEAM CASTOR, INRIA Sophia Antipolis, BP 9306902 Sophia Antipolis, France

3 KU Leuven, Department of Mechanical Engineering, 3001 Leuven, Belgium

4 TU Kaiserslautern, Chair for Scientific Computing, 67663 Kaiserslautern, Germany

(C) EDP Sciences, SMAI 2016 
transport modeling to find a design that reduces the heat load [4]. The results in [5] report an optimized shape design of the target structure obtained in less than ten times the computational cost of a single code evaluation. Later, similar optimal design methods were used in [6] to identify current configurations that meet the design goal using a perturbative model for magnetic field evaluations.

The identification of current configurations that meet certain design objectives is a standard problem in plasma equilibrium calculations. For control applications in tokamaks, it is often desirable to identify the coil currents that ensure the position and shape of the plasma core is close to the prescribed ones. Such problems are frequently solved with optimal design methods. We refer to [7] and [8] for examples of adjoint based optimization for tokamak control applications in the framework of sequential quadratic programming (SQP) [9]. Similar algorithms are used for real time plasma reconstruction codes [10-13], that identify the magnetic configuration that matches best with some observed measurement data.

The simplified magnetic field model used in [6] only approximately solves the plasma equilibrium problem (employing a vacuum approximation for additional external currents) and therefore limits the applicability towards magnetic configuration design. This paper deals with the incorporation of a more consistent model, the Grad-Shafranov-Schlüter equation [14-16] describing the free boundary plasma equilibrium (FBE), e.g. the magnetic field lines, for a given current configuration. We are using an implementation of the numerical methods described in $[8,17]$ to find approximate solutions of the FBE problem.

The objective of the optimization remains avoiding excessive heat peaks on the divertor targets. To achieve this objective with adjoint based optimization methods, an automated coupling is needed between the FBE code and the plasma edge transport code. However, the latter typically uses formulations in coordinate systems that are aligned with magnetic field lines. For this alignment, a transformation from an absolute spatial coordinate system to an orthogonal coordinate system aligned with the magnetic field lines has to be used. Such coordinate transformations allow to work with discretizations on structured grids while keeping the numerical diffusion sufficiently low. The generation of the structured grid itself often demands significant user input and is therefore a bottleneck when automating the simulation sequence, as has been discussed in [18]. We will therefore focus in this work on details of the automated coupling, such as an adaptive mesh refinement procedure for the FBE solver, in order to accurately characterize the sensitive region around the saddle point. To facilitate the automation, all models are integrated in a MATLAB framework.

The paper is organized as follows. In the first section the optimization problem for optimal magnetic configuration design is introduced. In section 2, an efficient and practical approach to calculate the sensitivity of the cost function is presented. Subsequently, in section 3, the model equations for the FBE, the coordinate transformation and the transport in plasma edge are introduced. We will mostly refer to literature for details about the numerical approximation of these model equations and elaborate only the details related to the numerical computation of the overall cost function. Finally, in section 4, the discussed method is applied to a realistic WEST case and validated.

\section{Introducing AN OPtimal DESIGN SETting}

The design problem that will be tackled is to find the best current configuration in the coils with respect to target heat loading. The first step to obtain an optimal design consists of reformulating the design problem into a mathematical optimization problem with constraints:

$$
\begin{array}{rl}
\min _{\boldsymbol{\varphi} \in \Phi_{a d}, \mathbf{q}} & \mathcal{I}(\boldsymbol{\varphi}, \mathbf{q}) \\
\text { s.t. } & \boldsymbol{c}(\boldsymbol{\varphi}, \mathbf{q})=0,
\end{array}
$$

where we make the distinction between two kinds of variables. The first are the control or design variables $\varphi$, which are the independent variables that need to be optimized. The second are the state variables $\mathbf{q}$, which 
are dependent variables that can be uniquely defined from the control variables, using the constraint model equation $\boldsymbol{c}(\boldsymbol{\varphi}, \mathbf{q})=0$. The scalar-valued objective $\mathcal{I}(\boldsymbol{\varphi}, \mathbf{q})$ represents the design goal.

In the search for a magnetic configuration for optimal target heat loading, the constraint consists of three components $\boldsymbol{c}=\left(c_{\mathrm{eq}}, \boldsymbol{c}_{\mathrm{ct}}, \boldsymbol{c}_{\mathrm{pe}}\right)^{T}$. The first component $c_{\mathrm{eq}}\left(\boldsymbol{\varphi}, q_{\mathrm{eq}}\right)$ is governed by the FBE equations [14-16]. Here, $\boldsymbol{\varphi}$ is a vector containing the coil currents to be optimized. The constraint $c_{\mathrm{eq}}\left(\boldsymbol{\varphi}, q_{\mathrm{eq}}\right)=0$ relates these coil currents $\boldsymbol{\varphi}$ to the poloidal magnetic flux $q_{\mathrm{eq}}$. The second constraint component $\boldsymbol{c}_{\mathrm{ct}}\left(q_{\mathrm{eq}}, \boldsymbol{q}_{\mathrm{ct}}\right)=0$ characterizes the coordinate transformation that is typically used for solving the plasma edge transport equations, relating theoretically the poloidal magnetic flux to the continuous metric coefficient fields $\boldsymbol{q}_{\mathrm{ct}}$. These metric coefficient fields govern the transformation of the Cartesian coordinate system to the field aligned curvilinear coordinate system. The third component $\boldsymbol{c}_{\mathrm{pe}}\left(\boldsymbol{q}_{\mathrm{ct}}, \boldsymbol{q}_{\mathrm{pe}}\right)$ contains the plasma edge transport equations, which relate these metric coefficients to the plasma state variables $\boldsymbol{q}_{\text {pe }}$, such as the plasma density and temperature.

As the envisaged cost functional can be determined from geometrical and plasma variables only, the optimization formulation now becomes

$$
\begin{aligned}
\underset{\boldsymbol{\varphi} \in \Phi_{a d}, q_{\mathrm{eq}}, \boldsymbol{q}_{\mathrm{ct}}, \boldsymbol{q}_{\mathrm{pe}}}{ } & \mathcal{I}\left(\boldsymbol{\varphi}, \boldsymbol{q}_{\mathrm{ct}}, \boldsymbol{q}_{\mathrm{pe}}\right) \\
\text { s.t. } & c_{\mathrm{eq}}\left(\boldsymbol{\varphi}, q_{\mathrm{eq}}\right)=0, \\
& \boldsymbol{c}_{\mathrm{ct}}\left(q_{\mathrm{eq}}, \boldsymbol{q}_{\mathrm{ct}}\right)=0, \\
& \boldsymbol{c}_{\mathrm{pe}}\left(\boldsymbol{q}_{\mathrm{ct}}, \boldsymbol{q}_{\mathrm{pe}}\right)=0 .
\end{aligned}
$$

The objective functional from [18] is adopted. This equals

$$
\mathcal{I}\left(\boldsymbol{\varphi}, \boldsymbol{q}_{\mathrm{ct}}, \boldsymbol{q}_{\mathrm{pe}}\right)=\frac{1}{2} \lambda_{Q} \int_{S_{t}}\left(Q_{\perp}-Q_{d, t}\right)^{2} d \boldsymbol{\sigma}+\frac{1}{2} \lambda_{\varphi} \sum_{i} \varphi_{i}^{2}
$$

where the surface integral with elementary surface vector $d \boldsymbol{\sigma}$ integrates over the target surface area $S_{t}$. $Q_{\perp}$ is the heat flux density perpendicular to the target surface and $Q_{d, t}$ a desirable spatially constant heat flux profile. The second term is a Tikhonov regularization term, which physically penalizes excessive Joule losses. $\lambda_{Q}$ and $\lambda_{\varphi}$ are both a normalization and a weighting factor for their corresponding terms.

\section{A PROBLEM ADAPTED EFFICIENT COMPUTATION OF THE OBJECTIVE GRADIENT}

We assume that the objective function $\mathcal{I}(\boldsymbol{\varphi}, \mathbf{q})$ and the constraints $\boldsymbol{c}(\boldsymbol{\varphi}, \mathbf{q})=0$ are continuously Fréchetdifferentiable and we have suitable Hilbert spaces for the state space, the design space, and their Cartesian product space. The solution of the constrained optimization problem (1) can then be obtained using a gradient based optimization algorithm [9]. This class of methods finds the direction of each optimization step based on

evaluations of the gradient $\nabla \hat{\mathcal{I}}(\varphi)$ of the reduced objective functional $\hat{\mathcal{I}}(\boldsymbol{\varphi}):=\mathcal{I}(\boldsymbol{\varphi}, \mathbf{q}(\boldsymbol{\varphi}))$, where we assume that $\partial \mathbf{c} / \partial \mathbf{q}$ is always invertible, i.e. the model is always solvable with respect to $\mathbf{q}$ and where the gradient operator is defined as the Riesz-representation of the Fréchet-derivative $\hat{\mathcal{I}}^{\prime}$. The directional derivative of $\hat{\mathcal{I}}$ in the direction of a control variable perturbation $\delta \varphi$ evaluates as

$$
\hat{\mathcal{I}}^{\prime}(\boldsymbol{\delta} \varphi) \equiv\langle\nabla \hat{\mathcal{I}}, \boldsymbol{\delta} \varphi\rangle=\left\langle\partial_{\boldsymbol{\varphi}} \mathcal{I}(\boldsymbol{\varphi}, \mathbf{q}), \boldsymbol{\delta} \boldsymbol{\varphi}\right\rangle+\left\langle\partial_{\mathbf{q}} \mathcal{I}(\boldsymbol{\varphi}, \mathbf{q}), \partial_{\boldsymbol{\varphi}} \mathbf{q}(\boldsymbol{\varphi}) \boldsymbol{\delta} \boldsymbol{\varphi}\right\rangle
$$

where $\langle\cdot, \cdot\rangle$ denotes suitable dual-primal pairings. If combined with an appropriate line search algorithm, gradient based optimization algorithms offer a guaranteed descent of the objective. Differentiating the constraints $\boldsymbol{c}(\boldsymbol{\varphi}, \mathbf{q}(\boldsymbol{\varphi}))=0$ in direction $\boldsymbol{\delta} \boldsymbol{\varphi}$ yields that the sensitivity $\partial_{\boldsymbol{\varphi}} \mathbf{q}(\boldsymbol{\varphi}) \boldsymbol{\delta} \boldsymbol{\varphi}$ of $\mathbf{q}(\boldsymbol{\varphi})$ in direction $\boldsymbol{\delta} \boldsymbol{\varphi}$ is the solution to

$$
\partial_{\mathbf{q}} c(\varphi, \mathbf{q}) \partial_{\varphi} \mathbf{q}(\varphi) \delta \varphi=-\partial_{\varphi} c(\varphi, \mathbf{q}) \delta \varphi
$$


The evaluation of $\partial_{\boldsymbol{\varphi}} \mathbf{q}(\boldsymbol{\varphi}) \boldsymbol{\delta} \boldsymbol{\varphi}$ via (5) can be expensive if the control variable vector $\boldsymbol{\varphi}$ has a large number of components. Hence, care has to be taken for these methods that gradient evaluations do not dominate the total computational cost.

Adjoint sensitivity calculations offer an attractive alternative to the direct solution of equations (4) and (5) and a gradient evaluation cost independent of the amount of control variables [19]. One possible derivation is based on the Lagrangian

$$
\mathcal{L}\left(\boldsymbol{\varphi}, \mathbf{q}, \mathbf{q}^{*}\right)=\mathcal{I}(\boldsymbol{\varphi}, \mathbf{q})+\left\langle\mathbf{q}^{*}, \boldsymbol{c}(\boldsymbol{\varphi}, \mathbf{q})\right\rangle
$$

with $\mathbf{q}^{*}$ the so-called Lagrange multipliers or "adjoint variables". Then, requiring that the derivatives of the Lagrangian with respect to its arguments equals zero leads to the Karush-Kuhn-Tucker (KKT) conditions of the optimization problem (1). Differentiation with respect to the adjoint and state variables leads to the state equations $\boldsymbol{c}(\boldsymbol{\varphi}, \mathbf{q})=0$ and to the so-called adjoint equations

$$
\left\langle\mathbf{q}^{*}, \partial_{\mathbf{q}} \boldsymbol{c}(\boldsymbol{\varphi}, \mathbf{q}) \delta \mathbf{q}\right\rangle=-\left\langle\partial_{\mathbf{q}} \mathcal{I}(\boldsymbol{\varphi}, \mathbf{q}), \delta \mathbf{q}\right\rangle \quad \forall \delta \mathbf{q}
$$

respectively. The gradient $\nabla \hat{\mathcal{I}}$ is then given by

$$
\nabla \hat{\mathcal{I}}=\partial_{\boldsymbol{\varphi}} \mathcal{L}\left(\boldsymbol{\varphi}, \mathbf{q}, \mathbf{q}^{*}\right)=\partial_{\boldsymbol{\varphi}} \mathcal{I}(\boldsymbol{\varphi}, \mathbf{q})+\left(\partial_{\boldsymbol{\varphi}} \boldsymbol{c}(\boldsymbol{\varphi}, \mathbf{q})\right)^{*} \mathbf{q}^{*}
$$

where $\mathbf{q}$ and $\mathbf{q}^{*}$ are the solutions of $c(\boldsymbol{\varphi}, \mathbf{q})=0$ and $(7)$, respectively, and ${ }^{*}$ represents the adjoint operator with respect to the dual-primal pairing $\langle\cdot, \cdot\rangle$. Equating (8) to zero leads to the so-called design equation of the optimization problem (1).

Given that the plasma edge transport simulation presented in this paper easily takes several hours on a single workstation (for the WEST case considered further), adjoint methods provide a reasonable effiency gain already at a small number of control variable degrees of freedom. If one wishes to include a more elaborated multispecies transport code such as B2-EIRENE, CPU-time of a single simulation sharply increases and might range up to a year (parallized over a cluster) for simulation of power plant relevant conditions. It is clear that for design or sensitivity studies with the latter code adjoint gradients are indispensable.

For our optimization problem (2) where $\mathbf{q}=\left(q_{\mathrm{eq}}, \boldsymbol{q}_{\mathrm{ct}}, \boldsymbol{q}_{\mathrm{pe}}\right)^{T}$, the gradient (8) of the reduced objective function is given by

$$
\nabla \hat{\mathcal{I}}=\partial_{\boldsymbol{\varphi}} \mathcal{I}\left(\boldsymbol{\varphi}, \boldsymbol{q}_{\mathrm{ct}}, \boldsymbol{q}_{\mathrm{pe}}\right)+\left(\partial_{\boldsymbol{\varphi}} c_{\mathrm{eq}}\left(\boldsymbol{\varphi}, q_{\mathrm{eq}}\right)\right)^{*} q_{\mathrm{eq}}^{*},
$$

where the Lagrange multiplier $\mathbf{q}^{*}=\left(q_{\mathrm{eq}}^{*}, \boldsymbol{q}_{\mathrm{ct}}^{*}, \boldsymbol{q}_{\mathrm{pe}}^{*}\right)$ is obtained by solving the adjoint equations

$$
\begin{array}{rlr}
\left\langle q_{\mathrm{eq}}^{*}, \partial_{q_{\mathrm{eq}}} c_{\mathrm{eq}}\left(\boldsymbol{\varphi}, q_{\mathrm{eq}}\right) \delta q_{\mathrm{eq}}\right\rangle+\left\langle\boldsymbol{q}_{\mathrm{ct}}^{*}, \partial_{q_{\mathrm{eq}}} \boldsymbol{c}_{\mathrm{ct}}\left(q_{\mathrm{eq}}, \boldsymbol{q}_{\mathrm{ct}}\right) \delta q_{\mathrm{eq}}\right\rangle & =0 & \forall \delta q_{\mathrm{eq}} \\
\left.\left\langle\boldsymbol{q}_{\mathrm{ct}}^{*}, \partial_{\boldsymbol{q}_{\mathrm{ct}}} \boldsymbol{c}_{\mathrm{ct}}\left(q_{\mathrm{eq}}, \boldsymbol{q}_{\mathrm{ct}}\right)\right) \boldsymbol{\delta} \boldsymbol{q}_{\mathrm{ct}}\right\rangle+\left\langle\boldsymbol{q}_{\mathrm{pe}}^{*}, \partial_{\boldsymbol{q}_{\mathrm{ct}}} \boldsymbol{c}_{\mathrm{pe}}\left(\boldsymbol{q}_{\mathrm{ct}}, \boldsymbol{q}_{\mathrm{pe}}\right) \boldsymbol{\delta} \boldsymbol{q}_{\mathrm{ct}}\right\rangle=-\left\langle\partial_{\boldsymbol{q}_{\mathrm{ct}}} \mathcal{I}\left(\boldsymbol{\varphi}, \boldsymbol{q}_{\mathrm{ct}}, \boldsymbol{q}_{\mathrm{pe}}\right), \boldsymbol{\delta} \boldsymbol{q}_{\mathrm{ct}}\right\rangle & \forall \boldsymbol{q}_{\mathrm{ct}}, \\
\left\langle\boldsymbol{q}_{\mathrm{pe}}^{*}, \partial_{\boldsymbol{q}_{\mathrm{pe}}} \boldsymbol{c}_{\mathrm{pe}}\left(\boldsymbol{q}_{\mathrm{ct}}, \boldsymbol{q}_{\mathrm{pe}}\right) \boldsymbol{\delta} \boldsymbol{q}_{\mathrm{pe}}\right\rangle=-\left\langle\partial_{\boldsymbol{q}_{\mathrm{pe}}} \mathcal{I}\left(\boldsymbol{\varphi}, \boldsymbol{q}_{\mathrm{ct}}, \boldsymbol{q}_{\mathrm{pe}}\right), \boldsymbol{\delta} \boldsymbol{q}_{\mathrm{pe}}\right\rangle & \forall \boldsymbol{\delta} \boldsymbol{q}_{\mathrm{pe}}
\end{array}
$$

Evaluation of the gradient $\nabla \hat{\mathcal{I}}$ of the reduced objective functional thus involves solving first successively the constraint equations for $\mathbf{q}(\boldsymbol{\varphi})$, after which the Lagrange multiplier $\mathbf{q}^{*}$ is obtained from solving (10). Remark that the adjoint equations in (10) should be solved in reversed order compared to the forward constraint equations. Hence, if we want to use a fully adjoint method to compute the gradient of our reduced objective functional we need to have explicit expressions of the derivatives of $\boldsymbol{c}_{\mathrm{pe}}, \boldsymbol{c}_{\mathrm{ct}}, c_{\mathrm{eq}}$, and $\mathcal{I}$ with respect to the control $\boldsymbol{\varphi}$ and state variables $q_{\mathrm{eq}}, \boldsymbol{q}_{\mathrm{ct}}$, and $\boldsymbol{q}_{\mathrm{pe}}$. The derivatives $\partial_{\boldsymbol{\varphi}} c_{\mathrm{eq}}\left(\boldsymbol{\varphi}, q_{\mathrm{eq}}\right)$ and $\partial_{q_{\mathrm{eq}}} c_{\mathrm{eq}}\left(\boldsymbol{\varphi}, q_{\mathrm{eq}}\right)$ of the equilibrium problem are available both for the continuous as well as for the discretized problem [8]. Also the derivative $\partial_{\boldsymbol{q}_{\mathrm{pe}}} \boldsymbol{c}_{\mathrm{pe}}\left(\boldsymbol{q}_{\mathrm{ct}}, \boldsymbol{q}_{\mathrm{pe}}\right)$ was given explicitly in [20]. However, it's rather cumbersome to find the analytic expressions for the derivatives $\partial_{\boldsymbol{q}_{\mathrm{ct}}} \boldsymbol{c}_{\mathrm{ct}}\left(\boldsymbol{q}_{\mathrm{ct}}, q_{\mathrm{eq}}\right)$ and $\partial_{q_{\mathrm{eq}}} \boldsymbol{c}_{\mathrm{ct}}\left(\boldsymbol{q}_{\mathrm{ct}}, q_{\mathrm{eq}}\right)$ of the coordinate transformation. Therefore, a pragmatic approach, combining adjoint methods with finite differences, is adopted to avoid these difficulties [6]. This approach exploits the fact that the computational costs related to the numerical solution of the FBE problem $c_{\mathrm{eq}}\left(\boldsymbol{\varphi}, q_{\mathrm{eq}}\right)=0$ and coordinate transformation $c_{\mathrm{ct}}\left(q_{\mathrm{eq}}, \boldsymbol{q}_{\mathrm{ct}}\right)=0$ are at least three orders of magnitude smaller than the cost associated to the plasma edge transport computation. 
We eliminate formally $q_{\mathrm{eq}}$ and $\boldsymbol{q}_{\mathrm{ct}}$ and introduce

$$
\widetilde{\mathcal{I}}\left(\boldsymbol{\varphi}, \boldsymbol{q}_{\mathrm{pe}}\right):=\mathcal{I}\left(\boldsymbol{\varphi}, \boldsymbol{q}_{\mathrm{ct}}\left(q_{\mathrm{eq}}(\boldsymbol{\varphi})\right), \boldsymbol{q}_{\mathrm{pe}}\right)
$$

and

$$
\widetilde{\boldsymbol{c}}\left(\boldsymbol{\varphi}, \boldsymbol{q}_{\mathrm{pe}}\right):=\boldsymbol{c}_{\mathrm{pe}}\left(\boldsymbol{q}_{\mathrm{ct}}\left(q_{\mathrm{eq}}(\boldsymbol{\varphi})\right), \boldsymbol{q}_{\mathrm{pe}}\right),
$$

where $\boldsymbol{q}_{\mathrm{ct}}\left(q_{\mathrm{eq}}\right)$ and $q_{\mathrm{eq}}(\boldsymbol{\varphi})$ are defined by $\boldsymbol{c}_{\mathrm{ct}}\left(q_{\mathrm{eq}}, \boldsymbol{q}_{\mathrm{ct}}\left(q_{\mathrm{eq}}\right)\right)=0$ and $c_{\mathrm{eq}}\left(\boldsymbol{\varphi}, q_{\mathrm{eq}}(\boldsymbol{\varphi})\right)=0$, respectively. With this notation, the constrained optimization problem (2) is equivalent to

$$
\begin{aligned}
\min _{\boldsymbol{\varphi} \in \Phi_{a d}, \boldsymbol{q}_{\mathrm{pe}}} & \tilde{\mathcal{I}}\left(\boldsymbol{\varphi}, \boldsymbol{q}_{\mathrm{pe}}\right) \\
\text { s.t. } & \tilde{\boldsymbol{c}}\left(\boldsymbol{\varphi}, \boldsymbol{q}_{\mathrm{pe}}\right)=0 .
\end{aligned}
$$

Hence, the gradient of the reduced objective function writes as

$$
\hat{\mathcal{I}}^{\prime}(\boldsymbol{\delta} \varphi)=\left\langle\partial_{\boldsymbol{\varphi}} \tilde{\mathcal{I}}\left(\boldsymbol{\varphi}, \boldsymbol{q}_{\mathrm{pe}}\right), \boldsymbol{\delta} \boldsymbol{\varphi}\right\rangle+\left\langle\boldsymbol{q}_{\mathrm{pe}}^{*}, \partial_{\boldsymbol{\varphi}} \widetilde{\boldsymbol{c}}\left(\boldsymbol{\varphi}, \boldsymbol{q}_{\mathrm{pe}}\right) \boldsymbol{\delta} \boldsymbol{\varphi}\right\rangle
$$

where now the Lagrange multiplier $\boldsymbol{q}_{\mathrm{pe}}^{*}$ solves the adjoint equation

$$
\left(\partial_{\boldsymbol{q}_{\mathrm{p}}} \widetilde{\boldsymbol{c}}\left(\boldsymbol{\varphi}, \boldsymbol{q}_{\mathrm{pe}}\right)\right)^{*} \boldsymbol{q}_{\mathrm{pe}}^{*}=-\partial_{\boldsymbol{q}_{\mathrm{pe}}} \tilde{\mathcal{I}}\left(\boldsymbol{\varphi}, \boldsymbol{q}_{\mathrm{pe}}\right) .
$$

In (14) and (15) all the derivatives except for $\partial_{\boldsymbol{\varphi}} \widetilde{\boldsymbol{c}}\left(\boldsymbol{\varphi}, \boldsymbol{q}_{\mathrm{pe}}\right)$ and $\partial_{\boldsymbol{\varphi}} \widetilde{\mathcal{I}}\left(\boldsymbol{\varphi}, \boldsymbol{q}_{\mathrm{pe}}\right)$ are explicitly available. The derivatives $\partial_{\boldsymbol{q}_{\mathrm{p}}} \widetilde{\mathcal{I}}\left(\boldsymbol{\varphi}, \boldsymbol{q}_{\mathrm{pe}}\right)$ and $\partial_{\boldsymbol{q}_{\mathrm{pe}}} \widetilde{\boldsymbol{c}}\left(\boldsymbol{\varphi}, \boldsymbol{q}_{\mathrm{pe}}\right)$ can be retrieved in [20]. Hence, we will approximate (14) by a central finite difference approximation $\Delta_{\varepsilon} \hat{\mathcal{I}}(\varphi)$ :

$$
\hat{\mathcal{I}}^{\prime}(\boldsymbol{\delta} \varphi) \approx \Delta_{\varepsilon} \hat{\mathcal{I}}(\boldsymbol{\delta} \varphi):=\frac{\tilde{\mathcal{I}}\left(\boldsymbol{\varphi}+\varepsilon \boldsymbol{\delta} \boldsymbol{\varphi}, \boldsymbol{q}_{\mathrm{pe}}\right)-\tilde{\mathcal{I}}\left(\boldsymbol{\varphi}-\varepsilon \boldsymbol{\delta} \boldsymbol{\varphi}, \boldsymbol{q}_{\mathrm{pe}}\right)}{2 \varepsilon}+\left\langle\boldsymbol{q}_{\mathrm{pe}}^{*} \frac{\widetilde{\boldsymbol{c}}\left(\boldsymbol{\varphi}+\varepsilon \boldsymbol{\delta} \boldsymbol{\varphi}, \boldsymbol{q}_{\mathrm{pe}}\right)-\widetilde{\boldsymbol{c}}\left(\boldsymbol{\varphi}-\varepsilon \boldsymbol{\delta} \boldsymbol{\varphi}, \boldsymbol{q}_{\mathrm{pe}}\right)}{2 \varepsilon}\right\rangle,
$$

with $\varepsilon$ fixed. For the evaluation of $\hat{\mathcal{I}}(\varphi)$, we need to solve successively the nonlinear problems $c_{\mathrm{eq}}\left(\boldsymbol{\varphi}, q_{\mathrm{eq}}(\boldsymbol{\varphi})\right)=0$, $\boldsymbol{c}_{\mathrm{ct}}\left(q_{\mathrm{eq}}(\boldsymbol{\varphi}), \boldsymbol{q}_{\mathrm{ct}}\left(q_{\mathrm{eq}}(\boldsymbol{\varphi})\right)\right)=0$, and $\boldsymbol{c}_{\mathrm{pe}}\left(\boldsymbol{q}_{\mathrm{ct}}\left(q_{\mathrm{eq}}(\boldsymbol{\varphi})\right), \boldsymbol{q}_{\mathrm{pe}}(\boldsymbol{\varphi})\right)=0$ for $q_{\mathrm{eq}}(\boldsymbol{\varphi}), \boldsymbol{q}_{\mathrm{ct}}\left(q_{\mathrm{eq}}(\boldsymbol{\varphi})\right)$, and $\boldsymbol{q}_{\mathrm{pe}}\left(\boldsymbol{q}_{\mathrm{ct}}\left(q_{\mathrm{eq}}(\boldsymbol{\varphi})\right)\right)$. Then we can solve the adjoint equation (15) for the Lagrange multiplier $\boldsymbol{q}_{\mathrm{pe}}^{*}$. To finally approximate each component $\hat{\mathcal{I}}^{\prime}(\delta \varphi)$ of the gradient of the reduced objective function $\nabla \hat{\mathcal{I}}$, we solve successively the perturbed nonlinear problems $c_{\mathrm{eq}}\left(\boldsymbol{\varphi} \pm \varepsilon \boldsymbol{\delta} \boldsymbol{\varphi}, q_{\mathrm{eq}}(\boldsymbol{\varphi} \pm \varepsilon \boldsymbol{\delta} \boldsymbol{\varphi})\right)$ and $\boldsymbol{c}_{\mathrm{ct}}\left(q_{\mathrm{eq}}(\boldsymbol{\varphi} \pm \varepsilon \boldsymbol{\delta} \boldsymbol{\varphi}), \boldsymbol{q}_{\mathrm{ct}}\left(q_{\mathrm{eq}}(\boldsymbol{\varphi} \pm \varepsilon \boldsymbol{\delta} \boldsymbol{\varphi})\right)\right)$ for $q_{\mathrm{eq}}(\boldsymbol{\varphi} \pm \varepsilon \boldsymbol{\delta} \boldsymbol{\varphi})$ and $\boldsymbol{q}_{\mathrm{ct}}\left(q_{\mathrm{eq}}(\boldsymbol{\varphi} \pm \varepsilon \boldsymbol{\delta} \varphi)\right)$. Whereas a straightforward central difference calculation of sensitivities using a central difference approach as (25) would lead to $2 \cdot n_{\varphi}+1$ full simulations, the in parts adjoint method reduces this to $2 \cdot n_{\varphi}+1$ equilibrium simulations complemented with one forward and one adjoint plasma edge simulation, with $n_{\varphi}$ the number of design variables.

\section{Modeling Equations}

\subsection{Free boundary equilibrium computation}

The balance of pressure gradient and magnetic forces in the plasma core, the solenoidal condition for the magnetic field and Ampère's law yield for axisymmetric configurations the Grad-Shafranov-Schlüter equation, which solves

$$
-R \nabla \cdot\left(\frac{1}{\mu_{0} R^{2}} \nabla \psi\right)=R p^{\prime}(\psi)+\frac{1}{\mu_{0} R} f f^{\prime}(\psi)
$$

for the poloidal magnetic flux $\psi=\psi(R, Z)$, with $p$ the pressure and $f$ the $R$-scaled toroidal component of the magnetic field. $\nabla$ is the differential operator in the coordinates $(R, Z)$ and $\mu_{0}$ the magnetic permeability in vacuum. The Grad-Shafranov-Schlüter equation (17) is an equation that holds in the domain covered by the 
plasma and depends nonlinearly on the magnetic flux $\psi$. The boundary of the plasma domain either touches an interior point of the reactor wall (limiter configuration) or the boundary contains one or more saddle points of $\psi$ (divertor configuration). The saddle points of $\psi$ are called X-points of $\psi$. The plasma domain is the largest subdomain of the interior of the reactor bounded by a closed flux isoline and containing the magnetic axis. The magnetic axis is the point where $\psi$ has its global maximum in the interior of the reactor. In vacuum or non-conducting materials we have the equation

$$
-R \nabla \cdot\left(\frac{1}{\mu R^{2}} \nabla \psi\right)=0
$$

for the flux, while in the domains covered by the coils we have

$$
-R \nabla \cdot\left(\frac{1}{\mu R^{2}} \nabla \psi\right)=\frac{I}{S}
$$

with $\mu$ the magnetic permeability of the material ( $\mu_{0}$ in vacuum), $I$ being the current of the coil and $S$ its cross section. In practice we consider the magnetic permeability to be different from $\mu_{0}$ only in ferromagnetic materials, where $\mu$ has a nonlinear dependence on $\psi$ to account for saturation. The flux $\psi$ vanishes at $R=0$ and at infinity. We refer to $[8$, Section 2.1] for more details.

In theory, $p$ and $f$ in equation (17) can be found by augmenting this equation with a core transport model. Here, we assume that the current profile, i.e. the function $j: \psi \mapsto R p^{\prime}(\cdot)+\frac{1}{\mu_{0} R} f f^{\prime}(\cdot)$ is known as a function of the normalized poloidal flux $\bar{\psi}$ with $0 \leqslant \bar{\psi} \leqslant 1$. A frequent a priori model is

$$
j(\bar{\psi}, R)=\lambda\left(\frac{R}{R_{0}} \beta+(1-\beta) \frac{R_{0}}{R}\right)\left(1-\bar{\psi}^{\alpha}\right)^{\gamma},
$$

with $R_{0}$ the major radius of the vacuum chamber and $\alpha, \beta, \gamma \in \mathbb{R}$ given parameters. The variable $\lambda$ is determined by imposing that the total plasma current equals a predetermined value $I_{\mathrm{P}}$. We refer to [21] for a physical interpretation of these parameters.

We are solving this FBE problem by the numerical methods outlined in $[8,17]$. The poloidal flux is approximated by a finite dimensional function that is piecewise linear with respect to an unstructured triangular mesh. The equations (17)-(19) are discretized by a standard Galerkin method and the boundary conditions at infinity are incorporated using a boundary integral method. The resulting nonlinear algebraic equations are solved via Newton's method. Details can be found in [8, Section 3.1-4.3].

The generation of a structured grid for the discretization of the equation $\boldsymbol{c}_{\mathrm{pe}}\left(\boldsymbol{q}_{\mathrm{ct}}, \boldsymbol{q}_{\mathrm{pe}}\right)=0$, describing transport in the plasma edge, is a very delicate procedure that requires accurate approximations of poloidal and radial particle, momentum, and energy fluxes. In this context it is crucial to locate accurately the X-point position. Therefore, we included an adaptive refinement step, based on the longest edge bisection [22]. After each converged Newton iteration all triangles close to either 1) the X-point, 2) the boundary of the plasma domain or 3) both are marked for refinement, longest edge bisection is applied to generate a new triangular mesh with refined elements at the marked locations (see Figure 1). The refinement indexing is based on the distance in number of grid cells to ensure an accurate grid for the plasma edge calculations. The Newton method is next restarted to solve the FBE problem on this new mesh. The initial guess in the Newton iteration can be easily interpolated from the previous numerical solution on the coarser mesh and hence the Newton method converges in a couple of iterations. Given that the largest distortions of magnetic flux lines are situated near the magnetic field extrema, we will mainly focus our refinement efforts on the regions around X-points.

\subsection{A coordinate transformation for anisotropic plasma edge transport}

Models for transport in the edge of a plasma are typically solved in a magnetic field aligned poloidal coordinate system to avoid excessive numerical diffusion, while keeping a clear grid structure [3,23-27]. In figure 2 we sketch 

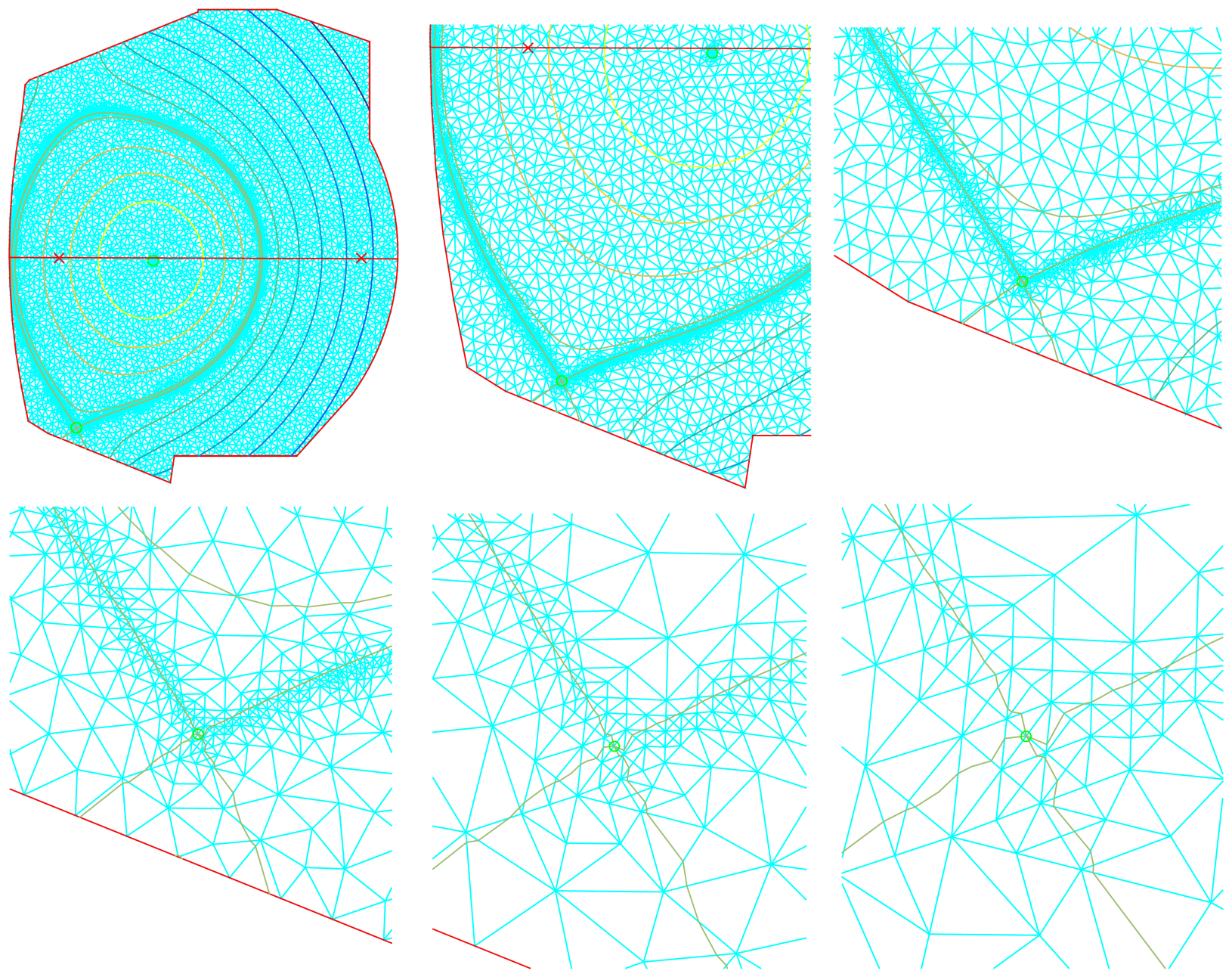

FiguRE 1. Example of a mesh (and 5 enlarged picture details) refined in the neighborhood of the plasma boundary. The contour lines indicate the poloidal flux.

the two mappings that are involved. The mapping

$$
\boldsymbol{G}_{\mathbf{1}}(\theta, r): \quad \mathbb{R}^{2} \rightarrow \mathbb{R}^{2}, \quad\left(\begin{array}{l}
r \\
\theta
\end{array}\right) \mapsto\left(\begin{array}{l}
R(\theta, r) \\
Z(\theta, r)
\end{array}\right)
$$

describes the transformation between poloidal section $(R, Z)$ of the basic cylindrical coordinate system $(R, Z, \phi)$ and the curvilinear coordinate system $(\theta, r)$ using the polodial magnetic flux $\psi$ as an input [28,29]. $\theta$ and $r$ are respectively the coordinates along and perpendicular to the isolines of the poloidal flux (see figure 2). The coordinate system $(\theta, r)$ is again an orthogonal coordinate system.

However, it is desirable that the solid target boundary is coinciding with one of the coordinate axes, so that a simple grid structure can be kept. Therefore, a second coordinate transformation

$$
\boldsymbol{G}_{\mathbf{2}}(x, y): \quad \mathbb{R}^{2} \rightarrow \mathbb{R}^{2}, \quad\left(\begin{array}{l}
x \\
y
\end{array}\right) \mapsto\left(\begin{array}{l}
\theta(x, y) \\
r(x, y)
\end{array}\right)
$$




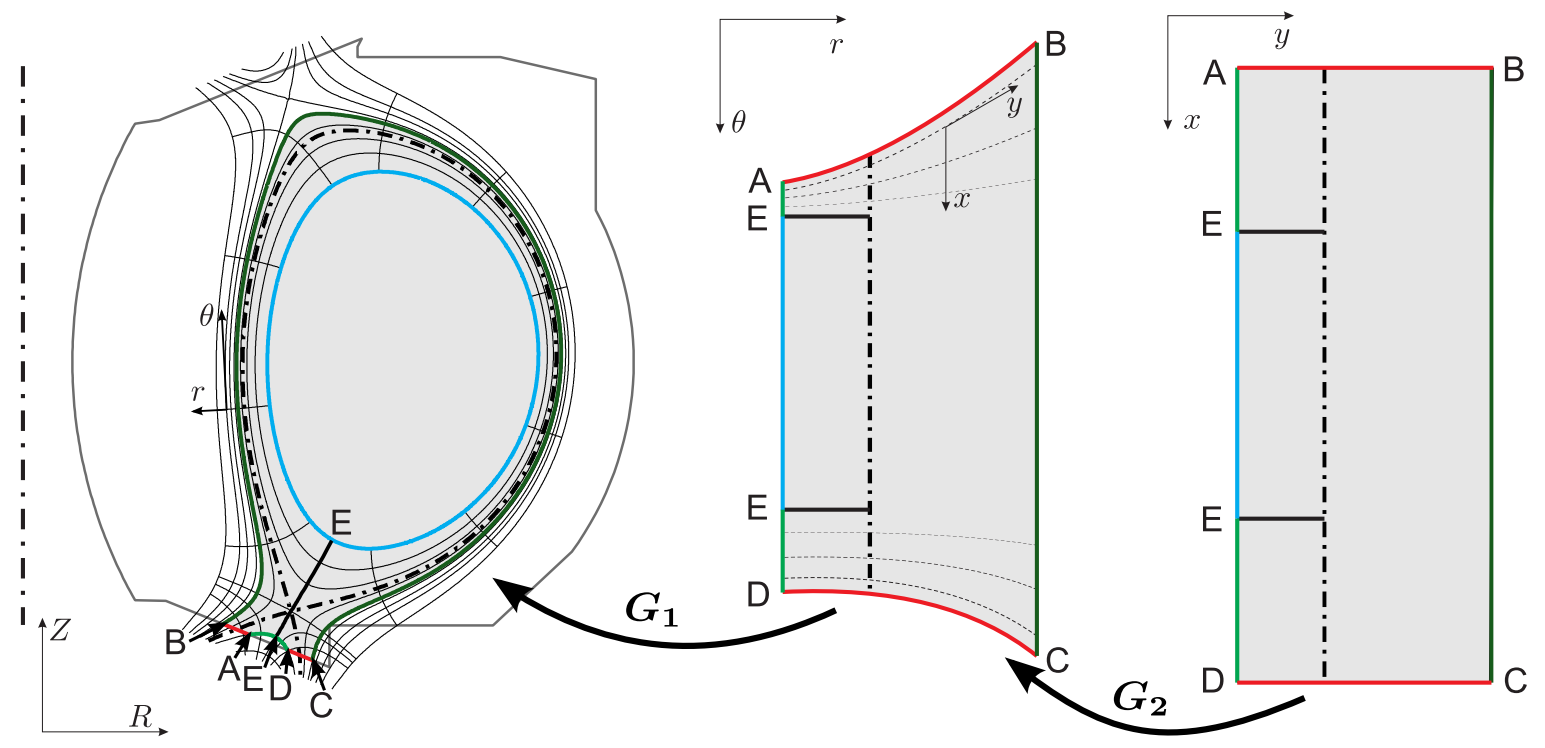

FiguRE 2. The grid generator mapping of the $(R, Z)$ coordinate system, via the orthogonal $(\theta, r)$ coordinate system, to the non-orthogonal $(x, y)$ coordinate system. Figure following Dekeyser [20].

is introduced that accommodates for the target geometry by relaxing the orthogonality constraint. The mapping $\boldsymbol{G}_{2}$ induces the transformation between the orthogonal coordinates system $(\theta, r)$ to the non-orthogonal curvilinear $(x, y)$ coordinate system, where $x$ is the coordinate along the isolines of poloidal magnetic flux lines(see a.o. [28, p. 88-89], [20, p. 71]). It is therefore clear that the freedom in this transformation entirely lies in the choice of the $y$ coordinate. Ideally, this choice maximizes the grid orthogonality, while smoothly adjusting for the solid geometry. Some algorithms directly pursuit this goal by using optimization algorithms [30]. Essentially, this is the most challenging part of the grid generator. Only recently, alternatives were proposed to impose boundary conditions for computational grids not aligned with the target structure [27,31], making the latter transformation redundant.

The two transformations $\boldsymbol{G}_{\mathbf{1}}$ and $\boldsymbol{G}_{\mathbf{2}}$ can be combined to find the transformation

$$
\boldsymbol{G}_{\mathrm{eq}}=\boldsymbol{G}_{1} \circ \boldsymbol{G}_{2}: \quad \mathbb{R}^{2} \rightarrow \mathbb{R}^{2}\left(\begin{array}{l}
x \\
y
\end{array}\right) \mapsto\left(\begin{array}{l}
R(x, y) \\
Z(x, y)
\end{array}\right) .
$$

and we can characterize this transformation by its metric coefficient matrix $g_{i j}$. In practice, only the metric coefficients $h_{x}=\left|\frac{\partial \boldsymbol{G}_{\mathrm{eq}}}{\partial x}\right|, h_{y}=\left|\frac{\partial \boldsymbol{G}_{\mathrm{eq}}}{\partial y}\right|$, angle $\alpha$ between the coordinate axis $\boldsymbol{e}_{x}$ and $\boldsymbol{e}_{y}$, and the Jacobian $J_{\text {eq }}=\sqrt{g}_{\text {eq }}=\sqrt{\operatorname{det}\left[g_{i j}\right]}$ of $(R, Z)$ with respect to $(x, y)$ are needed. Hence we have $\boldsymbol{q}_{\mathrm{ct}}=\left(h_{x}, h_{y}, \alpha, \sqrt{g}_{\mathrm{eq}}, b_{\theta}\right)^{T}$.

In discrete form, the transformation $\boldsymbol{G}_{\mathrm{eq}}$ is dealt with by a plasma edge grid generator. Isolines of the poloidal magnetic flux $\psi$ are traced using contour algorithms and each grid cell has two sides coinciding with two of these contours. Discrete metric coefficients are calculated from the cell geometries and represent cell widths in the direction of their coordinate axes. The Jacobian of the transformation is computed as the cell volume.

Although direct discretization of the plasma edge equations in the coordinate system will give rise to the smallest stencil size, this is not done as it would also result into an inaccurate radial (in the $\boldsymbol{e}_{r}$ direction) flux because of the strongly anisotropical transport. Therefore, the inverse transformation $\boldsymbol{G}_{2}^{-1}$ will be used to transform fluxes over iso- $x$ and iso- $y$ lines back to fluxes in the $(\theta, r)$ coordinate system and discretize the equations there. The computational stencil is then extended to compute correction terms resulting from the 
nonalignment of the radial lines with the coordinate axis $\boldsymbol{e}_{r}$ [32]. It is for this reason that the plasma edge equations in section 3.3 will be presented in the $(\theta, r)$-coordinate system, with the metric coefficients $h_{\theta}$, $h_{r}$, and the Jacobian $\sqrt{g}$ characterizing the transformation $\boldsymbol{G}_{\mathbf{1}}$.

\subsection{Plasma edge transport simulation}

All plasma edge models are a reduced form of the Braginskii [33] equations, augmented with a neutral model. For general notions on the Braginskii equations in a field aligned curvilinear coordinate system, we refer to [28]. In this paper we use a simplified model in comparison to the more advanced hybrid fluid-kinetic B2-EIRENE code [3] to somewhat reduce computational time and facilitate the demonstration of the optimal design procedure. The edge plasma transport model of [34] is used to model a single species plasma with ion mass $m$ and charge state $Z_{i}$ in a poloidal cross section of a toroidally symmetric tokamak for steady state. Particle and momentum conservation equations are solved for the ion density $n_{i}$ and ion parallel velocity $u_{\|}$. The neutral flow is modeled using a pressure diffusion equation to determine neutral pressure $p_{n}$. An internal energy equation is solved for a combined ion-electron-neutral temperature $T$. The vector of plasma state variables then becomes $\boldsymbol{q}_{\mathrm{pe}}=\left(n_{i}, u_{\|}, T, p_{n}\right)^{T}$. In the flux aligned coordinate system $(\theta, r)$ the plasma transport equations can be written succinctly as $\boldsymbol{c}_{\mathrm{pe}, \Omega}\left(\boldsymbol{q}_{\mathrm{ct}}, \boldsymbol{q}_{\mathrm{pe}}\right)=0$, with

$$
\begin{aligned}
\boldsymbol{c}_{\mathrm{pe}, \Omega}= & \frac{1}{\sqrt{g}} \frac{\partial}{\partial \theta}\left(\frac{\sqrt{g}}{h_{\theta}} C^{\theta}-\frac{\sqrt{g}}{h_{\theta}^{2}} D^{\theta} \frac{\partial \boldsymbol{q}_{\mathrm{pe}}}{\partial \theta}\right) \\
& +\frac{1}{\sqrt{g}} \frac{\partial}{\partial r}\left(\frac{\sqrt{g}}{h_{r}} C^{r}-\frac{\sqrt{g}}{h_{r}^{2}} D^{r} \frac{\partial \boldsymbol{q}_{\mathrm{pe}}}{\partial r}\right)-S,
\end{aligned}
$$

$C^{\theta}=\left(n_{i} u_{\theta}, m n_{i} u_{\theta} u_{\|}, \frac{5}{2}\left(1+Z_{i}\right) n_{i} u_{\theta} T, 0\right)^{T}$ and $C^{r}=0$ the poloidal and radial convective flux, and $u_{\theta}$ the ion poloidal velocity that is related to the parallel velocity $u_{\|}$by the magnetic pitch $b_{\theta}$, i.e. $u_{\theta}=b_{\theta} u_{\|}$. $D^{\theta}=\operatorname{diag}\left(0, \eta^{\theta}, \kappa^{\theta}, D_{p_{n}}\right)$ and

$$
D^{r}=\left(\begin{array}{cccc}
D_{i}^{r} & 0 & 0 & 0 \\
m D_{i}^{r} u_{\|} & \eta^{r} & 0 & 0 \\
\frac{5}{2}\left(1+Z_{i}\right) D_{i}^{r} T & 0 & \kappa^{r} & 0 \\
0 & 0 & 0 & D_{p_{n}}
\end{array}\right)
$$

are matrices containing respectively the poloidal and radial diffusive coefficients. The above equations are augmented with the equations for plasma and for neutral pressure, respectively $p=\left(1+Z_{i}\right) n_{i} T$ and $p_{n}=n_{n} T$. The sources $S\left(q_{\mathrm{ct}}, q_{\mathrm{pe}}\right)$ are defined as

$$
S=\left(\begin{array}{c}
n_{e} n_{n} K_{i}-n_{i} n_{e} K_{r} \\
-\frac{b_{\theta}}{h_{\theta}} \frac{\partial p}{\partial \theta}-m n_{i} n_{e} K_{r} u_{\|}-m n_{i} n_{n} K_{c x} u_{\|} \\
-E_{i} n_{e} n_{n} K_{i}-c_{z} n_{i} n_{e} L_{z} \\
n_{i} n_{e} K_{r}-n_{e} n_{n} K_{i}
\end{array}\right)
$$

with $E_{i}$ the energy lost by the plasma at ionization and $n_{e}=Z_{i} n_{i}$ the electron density. The contribution of the neutral velocity $u_{n \|}$ is left out in the ion momentum source as the neutral velocities resulting from this simple pressure-diffusion model tend to be unrealistically high. The impurity radiation is based on a prescribed, spatially constant impurity fraction $c_{z}$. Rate coefficients $K_{i}, K_{r}$ and $K_{c x}$ for electron impact ionization, radiative recombination and charge-exchange, respectively, as well as the radiative loss function $L_{z}$ of Carbon are approximated using the same analytical expressions as given in [4]. The isotropic neutral pressure diffusion coefficient $D_{p_{n}}$ is determined by a reformulation of the neutral momentum equation, where the latter is reduced to a balance between pressure gradient force and momentum source terms. Thus, the coefficient is given by

$$
D_{p_{n}}=\frac{1}{m\left(n_{i} K_{\mathrm{cx}}+n_{e} K_{\mathrm{i}}\right)} .
$$


Similar expressions can be found in a.o. [35]. Finally, the expression $\kappa_{n}=\chi_{n} p_{n} D_{p_{n}}$ is used for the neutral conductivity, while transverse plasma conductivity and ion viscosity are respectively given by $\kappa_{r}=n D_{\kappa}$ and $\eta_{r}=m n_{i} D_{i}^{r}$. The model equations $\boldsymbol{c}_{\mathrm{pe}, \Omega}=0$ are combined with appropriate boundary conditions, written formally as $\boldsymbol{c}_{\mathrm{pe}, \Sigma}=0$ to form the plasma model equations $\boldsymbol{c}_{\mathrm{pe}}=\left(\boldsymbol{c}_{\mathrm{pe}, \Omega}, \boldsymbol{c}_{\mathrm{pe}, \Sigma}\right)^{T}=0$, with $\Omega$ and $\Sigma$ respectively the domain of the plasma edge simulation and its boundary. In practice, these equations are discretized and solved using a finite volume method.

\section{VALidATION FOR A TEST CASE USING FINITE DIFFERENCE GRADIENT COMPUTATION}

The approximation (16) of the gradient of the objective functional derived in Section 2 is validated in this section using finite difference calculations

$$
\hat{\mathcal{I}}^{\prime}(\boldsymbol{\delta} \varphi) \approx \Delta_{\varepsilon}^{\mathrm{FD}} \hat{\mathcal{I}}(\varphi):=\frac{\hat{\mathcal{I}}(\varphi+\epsilon \delta \varphi)-\hat{\mathcal{I}}(\varphi-\epsilon \delta \varphi)}{2 \epsilon} .
$$

for the reduced objective function $\widehat{\mathcal{I}}(\varphi)$. The derivation of (16) follows mutatis mutandis for a discretized version of the optimal design problem (2) based on the methods mentioned in Section 3.

The validation is performed for a realistic test case that will be defined in subsection 4.1. The comparison itself is shown in subsection 4.2 .

\subsection{Set-up of the WEST test case}

Given the limitations of the plasma edge transport model, we choose a test case similar to the SOLEDGE2DEIRENE simulation discribed in [36] (The High power FAR configuration, with a puff of $1.1 \cdot 10^{21} \# / \mathrm{s}$ ). Two important differences in our model with respect to the latter must be noted. At the one hand, a kinetic code for neutrals is absent in our model and replaced by a fluid neutral model. At the other hand, a methodology to simulate up to the reactor wall, such as in [31] or [27], is absent in the radial (perpendicular to the magnetic flux lines) direction. Therefore, additional assumptions on boundary conditions and neutral fluid properties need to be made.

The magnetic equilibrium $\psi$ is obtained using the FBE code described in section 3.1, with $\alpha=1, \beta=1.5$, $\gamma=0.9, I_{p}=0.47 \mathrm{MA}$, and the coil currents as given in table 1 .

\begin{tabular}{|c||ccccccccccccccccc|}
\hline Conductor & 1 & 2 & 3 & 4 & 5 & 6 & 7 & 8 & 9 & 10 & 11 & 12 & 13 & 14 & 15 & 16 & 17 \\
\hline$I(\mathrm{kA})$ & 0 & 0 & 0 & 22.3 & -186.4 & -186.4 & -22.3 & 0 & 0 & 41.9 & 41.9 & 41.9 & 41.9 & 51.2 & 51.2 & 51.2 & 51.2 \\
\hline
\end{tabular}

TABLE 1. Values of the coil currents used in the FBE simulation. Conductor numbers corresponding to the numbers in figure 3 .

The plasma edge simulation is performed on a $210 \times 80$ grid, as given in red in figure 4 . In the parallel direction, plasma transport coefficients are set according to Braginskii [33]. Radial anomalous transport coefficients comprise a radial ion diffusivity $D_{i}^{r}=0.6 \mathrm{~m}^{2} \mathrm{~s}^{-1}$, a transverse ion viscosity coefficient $D_{\eta}=0.2 \mathrm{~m}^{2} \mathrm{~s}^{-1}$, a radial plasma conductivity coefficient $D_{\kappa}=D_{\kappa_{i}}+D_{\kappa_{e}}=4 \mathrm{~m}^{2} \mathrm{~s}^{-1}$ and a neutral conductivity coefficient $\chi_{n}=0.2$.

At the core boundary the input power $\left(Q_{\text {in }}=7.93 \mathrm{MW}\right)$ and the core density $\left(n_{i, c}=2.7 \cdot 10^{19} \mathrm{~m}^{-3}\right)$ are specified. For the neutrals a leakage condition proportional to the product of local neutral density and thermal speed [37] is given, with proportionality constant 0.167 . This proportionality constant was chosen to match the total neutral flux to the core region. $u_{\|}=0 \mathrm{~m}^{2} \mathrm{~s}^{-1}$ is assumed for the momentum equation at the core boundary. Sheath conditions are imposed at the divertor targets. At the outermost flux surfaces a radial decay length is assumed for plasma density $\left(\lambda_{n}=0.05 \mathrm{~m}\right)$ and temperature $\left(\lambda_{T}=0.3 \mathrm{~m}\right)$. All ions reaching wall and target domain boundaries are recycled as neutrals. Additionally, a smooth variable sticking fraction is used along the radial boundaries, so that the total pumped flux equals $7 \cdot 10^{20} \# / \mathrm{s}$, matching the SOLEDGE2D-EIRENE pumped flux. 


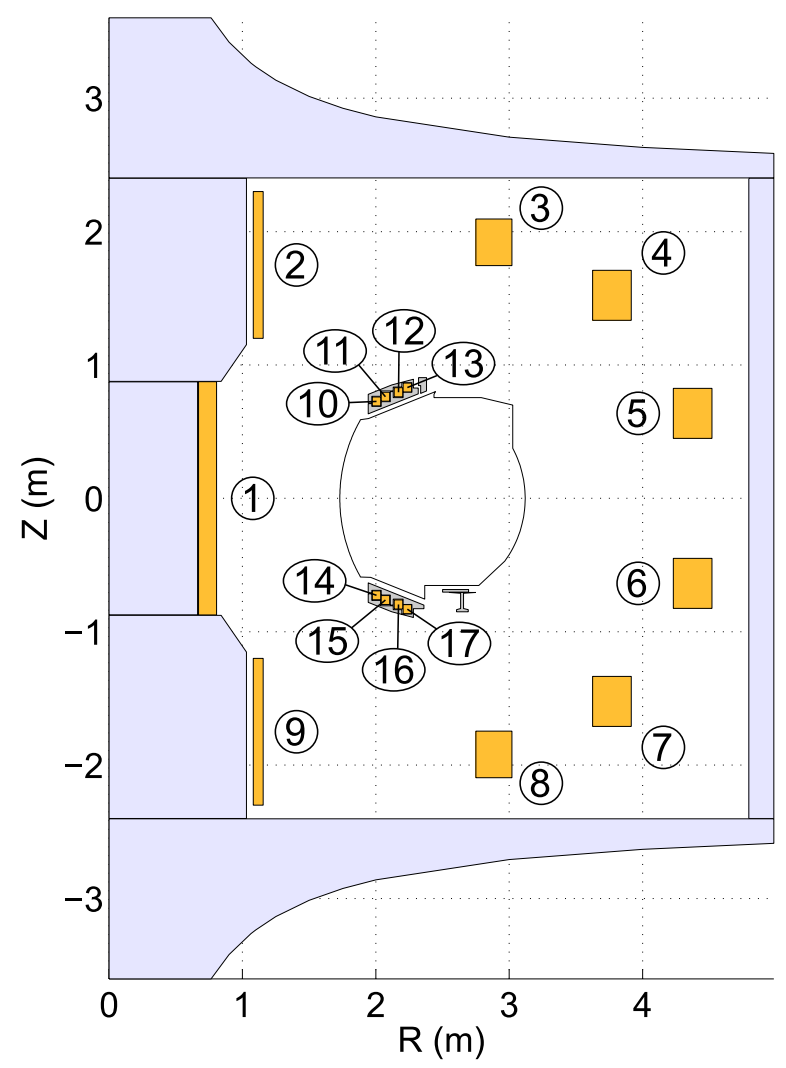

FIgURE 3. The components of the WEST case, as they enter the FBE computation. The vacuum vessel is indicated by the black line, coils are indicated in orange and numbered, iron structures are indicated in light grey and passive structures in darker grey.

\subsection{Results}

The design vector $\varphi$ for which we validate the gradient of the objective functional, consists exclusively of the shaping coil currents, i.e. $\varphi=\left[\begin{array}{lllll}I_{10} & I_{11} & \ldots & I_{17}\end{array}\right]^{T}$. The comparison of the two gradient computations is given in figure 5. As can be seen from this figure, the partially adjoint gradient is reasonably accurate and remains close to the "full" central difference calculations, although it slightly overestimates the value of the different gradient components. Especially for the first four components it can be seen that there is a systematic (but small) deviation. This deviation is due to the choice for a continuous adjoint (optimize-then-discretize) approach and is of the same order of magnitude than found in other work [20, p. 98]. However, the approaches are identical in the limit of infinitely fine grids, as the difference is situated solely in the discretization process.

In table 2 we finally provide the error

$$
\operatorname{Err}(\varepsilon)=\left|\Delta_{\varepsilon}^{\mathrm{FD}} \hat{\mathcal{I}}(\boldsymbol{\delta} \boldsymbol{\varphi})-\Delta_{\varepsilon} \hat{\mathcal{I}}(\boldsymbol{\delta} \boldsymbol{\varphi})\right|
$$




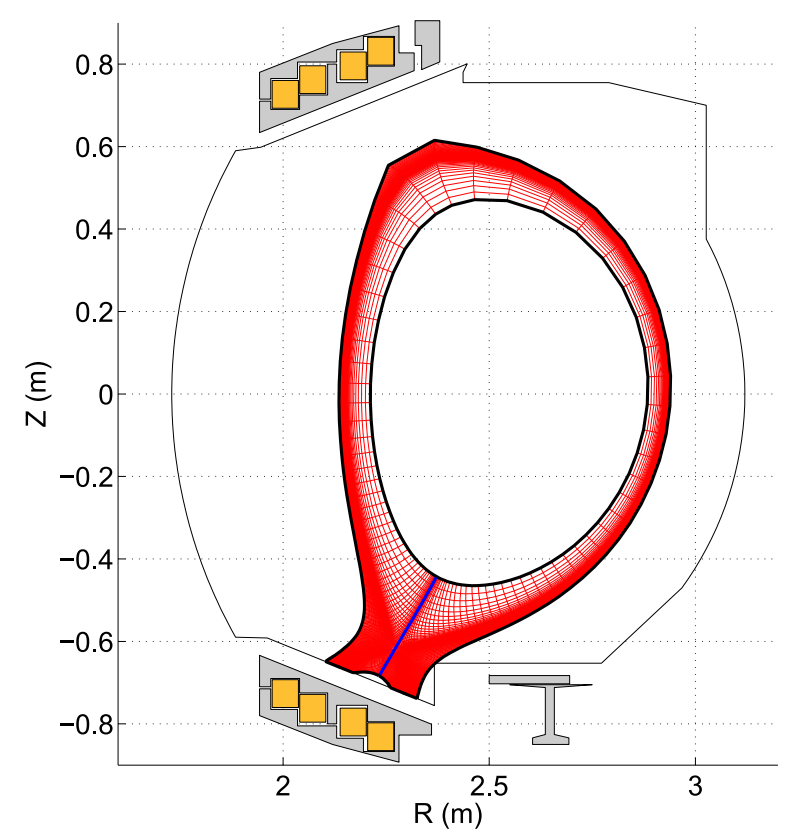

FIgURE 4. The plasma edge grid in the vacuum chamber. The boundaries are indicated by a tick black line. The blue lines indicate the grid cuts that are used to unfold the grid onto a topologically rectangular computational mesh.

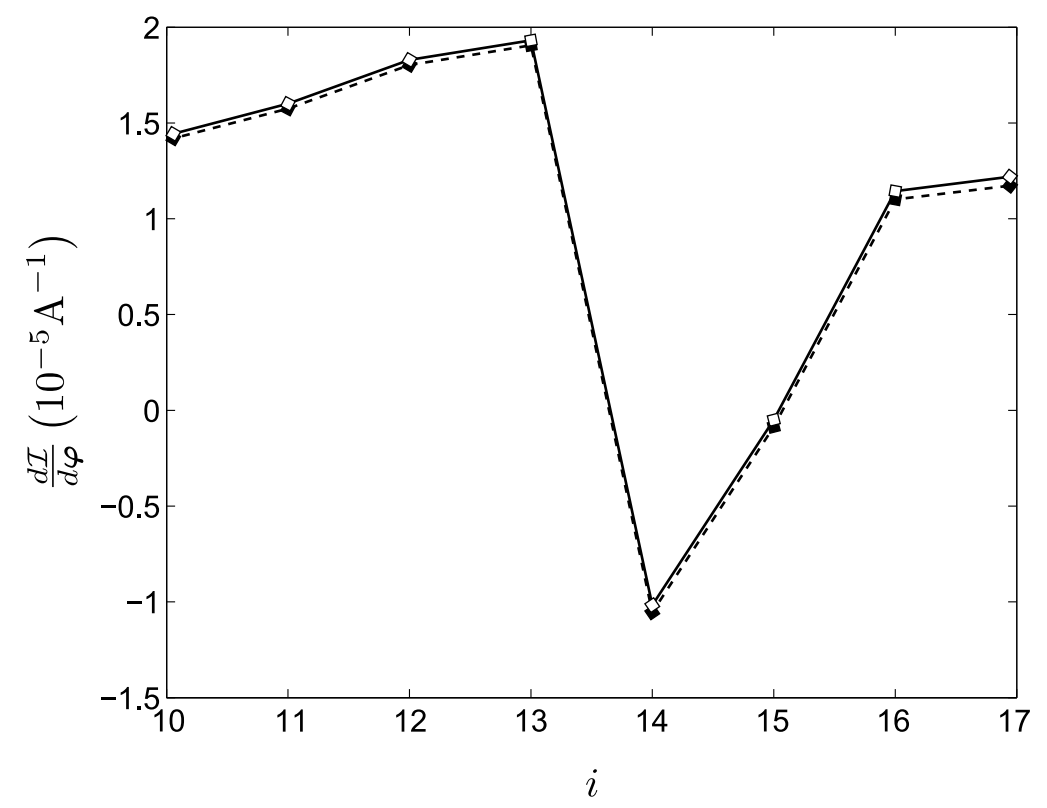

FiguRE 5. Evaluation of the design gradient $\nabla \hat{\mathcal{I}}=\left[\frac{d \mathcal{I}}{d I_{10}} \frac{d \mathcal{I}}{d I_{11}} \ldots \frac{d \mathcal{I}}{d I_{17}}\right]^{T}$, using: the approximations $\Delta_{\varepsilon}^{\mathrm{FD}} \hat{\mathcal{I}}(\boldsymbol{\delta} \boldsymbol{\varphi})$ defined in (25) (thick dashed line) and $\Delta_{\varepsilon} \hat{\mathcal{I}}(\boldsymbol{\delta} \boldsymbol{\varphi})$ as defined in (16) (thick solid line) with $\varepsilon=\varepsilon_{\mathrm{m}}^{\frac{1}{4}}$ and $\varepsilon_{\mathrm{m}}$ machine precision. 
of the two gradient approximations $\Delta_{\varepsilon}^{\mathrm{FD}} \hat{\mathcal{I}}(\boldsymbol{\delta} \boldsymbol{\varphi})$ and $\Delta_{\varepsilon} \hat{\mathcal{I}}(\boldsymbol{\delta} \boldsymbol{\varphi})$ as defined in (25) and (16) for different finite difference steps $\varepsilon$. One can see that for $\varepsilon$ different from $\varepsilon=\varepsilon_{m}^{\frac{1}{4}}$. The results depicted in figure 5 can thus further be improved by changing the step size $\epsilon$. Remark that apart from the cancellation and the truncation error, also the discretization error is influenced by the step $\epsilon$. Of course, each change in control variable causes a changed plasma edge grid. Therefore, a typical balance between truncation and cancellation errors as in many finite difference step studies is not necessarily to be expected here, as one can observe in table 2 .

\begin{tabular}{l|llllllll}
$\varepsilon$ & $I_{10}$ & $I_{11}$ & $I_{12}$ & $I_{13}$ & $I_{14}$ & $I_{15}$ & $I_{16}$ & $I_{17}$ \\
\hline$\varepsilon_{\mathrm{m}}^{\frac{1}{3}}$ & 0.0235 & 0.0254 & 0.0271 & 0.0267 & 0.0242 & 0.0252 & 0.0178 & 0.0094 \\
$\varepsilon_{\mathrm{m}}^{\frac{1}{4}}$ & 0.0236 & 0.0254 & 0.0271 & 0.0267 & 0.0376 & 0.0364 & 0.0431 & 0.0471 \\
$\varepsilon_{\mathrm{m}}^{\frac{1}{5}}$ & 0.0120 & 0.0136 & 0.0150 & 0.0144 & 0.0131 & 0.0171 & 0.0138 & 0.0130
\end{tabular}

TABLE 2. The absolute difference of the two gradient approximations $\Delta_{\varepsilon}^{\mathrm{FD}} \hat{\mathcal{I}}(\boldsymbol{\delta} \boldsymbol{\varphi})$ and $\Delta_{\varepsilon} \hat{\mathcal{I}}(\boldsymbol{\delta} \boldsymbol{\varphi})$ for different perturbations $\varepsilon$, with $\varepsilon_{\mathrm{m}}$ the machine precision.

As predicted, the plasma edge simulation dominated the computational cost and the gain of the in parts adjoint approach with respect to the central difference calculation was roughly the predicted factor $\left(2 \cdot n_{\varphi}+1\right) / 2 \approx$ $n_{\varphi}=8$. However, the gain slightly decreased for smaller finite difference step sizes. In this case, the perturbed plasma edge simulations can benefit more from using the reference simulation as a good initial solution.

\section{General COnClusions And Future Perspectives}

A sensitivity calculation is established that retrieves the influences of shaping coil changes on structure heat loads. For this purpose, a Free Boundary Equilibrium code and a plasma edge transport code were integrated in a code package to work in an automated fashion. It was pointed out that the specific transformation that plasma edge codes need to use, requires accurate magnetic flux contours near poloidal magnetic flux extrema. Therefore, an adaptive refinement procedure was set up for the equilibrium solver. Because of the significant computational costs of the plasma edge transport solver, an adjoint based sensitivity calculation was sought. Finding the equations that govern the coordinate transformation, which is represented by a grid generator in a discrete framework, however, appears to be rather cumbersome. Therefore, a more practical in parts adjoint sensitivity calculation is proposed as a reasonable compromise. The latter succeeds in keeping most of the computational cost reduction, while circumventing the difficulties with the coordinate transformation. This "partially" adjoint gradient calculation finally has been tested for a realistic WEST case and appears to match reasonably well with a finite difference sensitivity calculation. In future work, we plan to use these sensitivities for optimal design and attempt to use so-called one-shot methods [38] to reduce computational time for the entire design process even more.

\section{Acknowledgement}

The authors wish to thank Yannick Marandet and Hugo Bufferand for providing additional data on their WEST plasma edge transport simulations and for intensive support to set up the illustrative test case. We thank Eric Nardon for providing additional data on his WEST FBE solutions. The first author acknowledges support from OPTEC (OPTimization in Engineering Center of excellence KU Leuven), which is funded by the KU Leuven Research Council under grant no PFV/10/002.

\section{REFERENCES}

[1] A. Kukushkin, H. Pacher, V. Kotov, G. Pacher, and D. Reiter, "Finalizing the ITER divertor design: The key role of SOLPS modeling," Fusion Engineering and Design, vol. 86, pp. 2865-2873, Dec. 2011. 
[2] M. Kotschenreuther, P. M. Valanju, S. M. Mahajan, and J. C. Wiley, "On heat loading, novel divertors, and fusion reactors," Physics of Plasmas, vol. 14, no. 7, p. 072502, 2007.

[3] D. Reiter, M. Baelmans, and P. Börner, "The EIRENE and B2-EIRENE codes," Fusion Science and Technology, vol. 47, pp. 172-186, Feb 2005.

[4] W. Dekeyser, D. Reiter, and M. Baelmans, "Divertor Design through Shape Optimization," Contrib. Plasma Phys., vol. 52, pp. 544-549, June 2012.

[5] W. Dekeyser, D. Reiter, and M. Baelmans, "Divertor target shape optimization in realistic edge plasma geometry," Nuclear Fusion, vol. 54, no. 7, p. 073022, 2014.

[6] M. Blommaert, M. Baelmans, W. Dekeyser, N. Gauger, and D. Reiter, "A novel approach to magnetic divertor configuration design," Journal of Nuclear Materials, vol. 463, pp. 1220-1224, Aug. 2015.

[7] J. Blum, Numerical simulation and optimal control in plasma physics. Wiley/Gauthier-Villars, 1989.

[8] H. Heumann, J. Blum, C. Boulbe, B. Faugeras, G. Selig, J.-M. Ané, S. Brémond, V. Grandgirard, P. Hertout, and E. Nardon, "Quasi-static free-boundary equilibrium of toroidal plasma with CEDRES++: Computational methods and applications," Journal of Plasma Physics, vol. FirstView, pp. 1-35, 12015.

[9] J. Nocedal and S. Wright, Numerical Optimization. Springer, 2006.

[10] F. Hofmann and G. Tonetti, "Tokamak equilibrium reconstruction using Faraday rotation measurements," Nuclear Fusion, vol. 28 , no. 10, p. 1871, 1988.

[11] L. Lao, J. Ferron, R. Geoebner, W. Howl, H. St. John, E. Strait, and T. Taylor, "Equilibrium analysis of current profiles in Tokamaks," Nuclear Fusion, vol. 30, no. 6, p. 1035, 1990.

[12] P. Mc Carthy, P. Martin, and W. Schneider, "The CLISTE Interpretive Equilibrium Code," Tech. Rep. IPP Report 5/85, Max-Planck-Institut fur Plasmaphysik, 1999.

[13] J. Blum, C. Boulbe, and B. Faugeras, "Reconstruction of the equilibrium of the plasma in a tokamak and identification of the current density profile in real time," Journal of Computational Physics, vol. 231, no. 3, pp. 960 - 980, 2012.

[14] H. Grad and H. Rubin, "Hydromagnetic equilibria and force-free fields," Proceedings of the 2nd UN Conf. on the Peaceful Uses of Atomic Energy, vol. 31, p. 190, 1958.

[15] V. Shafranov, "On magnetohydrodynamical equilibrium configurations," Soviet Journal of Experimental and Theoretical Physics, vol. 6, p. 545, 1958.

[16] R. Lüst and A. Schlüter, "Axialsymmetrische magnetohydrodynamische Gleichgewichtskonfigurationen.," Z. Naturforsch. A, vol. 12 , pp. 850-854, 1957.

[17] J. Blum, J. Le Foll, and B. Thooris, "The self-consistent equilibrium and diffusion code SCED," Computer Physics Communications, vol. 24, pp. $235-254,1981$.

[18] M. Blommaert, W. Dekeyser, M. Baelmans, N. Gauger, and D. Reiter, "An Automated Approach to Magnetic Divertor Configuration Design," Nuclear Fusion, vol. 55, no. 013001, 2015.

[19] M. Hinze, R. Pinnau, M. Ulbrich, and S. Ulbrich, Optimization with PDE constraints, vol. 23 of Mathematical Modelling: Theory and Applications. New York: Springer, 2009.

[20] W. Dekeyser, D. Reiter, and M. Baelmans, Optimal Plasma Edge Configurations for Next-Step Fusion Reactors (Optimale plasmarand-configuraties voor nieuwe generatie fusiereactoren). PhD thesis, KU Leuven, 2014.

[21] J. Luxon and B. Brown, "Magnetic analysis of non-circular cross-section tokamaks," Nuclear Fusion, vol. 22, no. 6, p. 813, 1982.

[22] E. Bänsch, "Local mesh refinement in 2 and 3 dimensions," Impact Comput. Sci. Engrg., vol. 3, no. 3, pp. 181-191, 1991.

[23] R. Simonini, G. Corrigan, G. Radford, J. Spence, and A. Taroni, "Models and Numerics in the Multi-Fluid 2-D Edge Plasma Code EDGE2D/U," Contrib. Plasma Phys., vol. 34, pp. 368-373, Jan. 1994.

[24] S. Wiesen, "EDGE2D/EIRENE code interface report," tech. rep., Forschungszentrum Juelich, 2006.

[25] T. Rognlien, J. Milovich, M. Rensink, and G. Porter, "A fully implicit, time dependent 2-D fluid code for modeling tokamak edge plasmas," Journal of Nuclear Materials, vol. 196198, pp. 347-351, Dec. 1992.

[26] D. Stotler, C. Karney, M. Rensink, and T. Rognlien, "Coupling of Parallelized DEGAS 2 and UEDGE Codes," Contrib. Plasma Phys., vol. 40, pp. 221-226, June 2000.

[27] H. Bufferand, B. Bensiali, J. Bucalossi, G. Ciraolo, P. Genesio, P. Ghendrih, Y. Marandet, A. Paredes, F. Schwander, E. Serre, and P. Tamain, "Near wall plasma simulation using penalization technique with the transport code SolEdge2D-Eirene," Journal of Nuclear Materials, vol. 438, Supplement, pp. S445-S448, July 2013.

[28] M. Baelmans, Code Improvements and Applications of a Two-dimensional Edge Plasma Model for Toroidal Devices. PhD thesis, Departement Mechanica, Faculteit der Toegepaste Wetenschappen, Katholieke Universiteit Leuven, October 1993.

[29] W. D'haeseleer, W. Hitchon, J. Callen, and J. Shohet, Flux Coordinates and Magnetic Field Structure. Berlin: Springer-Verlag, 1991.

[30] R. Marchand and M. Dumberry, "CARRE: a quasi-orthogonal mesh generator for 2D edge plasma modelling," Computer Physics Communications, vol. 96, pp. 232-246, Aug. 1996.

[31] M. Baelmans, P. Börner, W. Dekeyser, and D. Reiter, "Tokamak plasma edge modelling including the main chamber wall," Nuclear Fusion, vol. 51, no. 8, p. 083023, 2011. 
[32] W. Dekeyser, D. Reiter, and M. Baelmans, "A one shot method for divertor target shape optimization," PAMM, vol. 14, no. 1, pp. 1017-1022, 2014.

[33] S. I. Braginskii, "Transport Processes in a Plasma," Reviews of Plasma Physics, vol. 1, p. 205, 1965.

[34] M. Baelmans, M. Blommaert, J. De Schutter, W. Dekeyser, and D. Reiter, "Efficient parameter estimation in 2D transport models based on an adjoint formalism," Plasma Physics and Controlled Fusion, vol. 56, p. 114009, 2014.

[35] M. E. Rensink, L. Lodestro, G. D. Porter, T. D. Rognlien, and D. P. Coster, "A Comparison of Neutral Gas Models for Divertor Plasmas," Contrib. Plasma Phys., vol. 38, pp. 325-330, Jan. 1998.

[36] H. Bufferand, J. Bucalossi, G. Ciraolo, N. Fedorczak, P. Ghendrih, R. Leybros, Y. Marandet, E. Serre, and P. Tamain, "Density Regimes and Heat Flux Deposition in the WEST Shallow Divertor Configuration," Contrib. Plasma Phys., vol. 54, pp. 378-382, June 2014.

[37] D. P. Coster, X. Bonnin, B. Braams, D. Reiter, R. Schneider, and the ASDEX Upgrade Team, "Simulation of the Edge Plasma in Tokamaks," Physica Scripta, vol. 2004, no. T108, pp. 7-13, 2004.

[38] S. Hazra and V. Schulz, "Simultaneous Pseudo-Timestepping for PDE-Model Based Optimization Problems," BIT Numerical Mathematics, vol. 44, no. 3, pp. 457-472, 2004.

This is an Open Access article distributed under the terms of the Creative Commons Attribution License (http://creativecommons.org/licenses/by/4.0), which permits unrestricted use, distribution, and reproduction in any medium, provided the original work is properly cited. 